Cuad. Invest. Filol., 43 (2017), 209-231. http://doi.org/10.18172/cif.3007

\title{
LA TRADUCCIÓN DEL DIALECTO EN LAS NARRACIONES DE ESCLAVOS: EL CASO DE THE GOOD LORD BIRD
}

\author{
Miguel Sanz Jiménez \\ Universidad Complutense de Madrid \\ miguelsanz@ucm.es
}

\begin{abstract}
RESUMEN: Este artículo explora la posibilidad de traducir al español la tercera novela de James McBride, The Good Lord Bird, y plantea una propuesta de traducción. Con el fin de ofrecer un texto en la lengua meta que reproduzca la variación lingüística del original y sus connotaciones, se estudian las estrategias para la traducción del dialecto en literatura y sus implicaciones sociales e ideológicas, que se aplican al caso particular del Black English y las narraciones de esclavos. También se consideran las posturas de varios traductólogos al respecto de la traducción del dialecto, así como las prácticas más extendidas en las versiones en español de las novelas pertenecientes a este género.

PALABRAS CLAVE: Black English, traducción del dialecto, James McBride, narraciones de esclavos, The Good Lord Bird, traducción literaria.
\end{abstract}

\section{TRANSLATING DIALECT IN SLAVE NARRATIVES: THE CASE OF THE GOOD LORD BIRD}

\begin{abstract}
This paper deals with the translation into Spanish of James McBride's third novel, The Good Lord Bird, and lays out a translation proposal. In order to obtain a text in the target language that reproduces the source's linguistic variation and its connotations, the strategies for translating literary dialect and its social and ideological implications are considered, specifically in the case of Black English and slave narratives. Translation scholars'views are also taken into account when it comes to translating dialect, together with certain examples of the Spanish renderings of some of the novels that belong to this particular genre.

KEYWORDS: Black English, Dialect Translation, James McBride, Slave Narratives, The Good Lord Bird, Literary Translation.
\end{abstract}

Recibido: 15/07/2016. Aceptado: 13/09/2016

\section{Introducción}

El objetivo de este artículo es considerar las posibilidades de traducir al español The Good Lord Bird, la novela del escritor estadounidense James McBride que 
se inscribe en el género de las narraciones de esclavos. Con este fin, el segundo apartado contextualiza la obra objeto de estudio para, a continuación, pasar a la problemática que supone la traducción del Black English en la novela. La cuarta sección de este trabajo revisa las posturas y estrategias de varios traductólogos con relación a la traducción del dialecto y las soluciones posibles a este escollo, que contrastan con la práctica editorial a la hora de publicar las narraciones de esclavos en castellano, pues, como se verá en el quinto apartado, se percibe el predominio de una estrategia en concreto que elimina cualquier tipo de variación lingüística presente en el texto fuente. Tras estas consideraciones teóricas y prácticas, se presenta una propuesta de traducción que distingue los dialectos presentes en The Good Lord Bird y se apoya en las reflexiones traductológicas antes enunciadas.

\section{The Good Lord Bird}

En 2013, James McBride, escritor, compositor, saxofonista y guionista estadounidense, publicó The Good Lord Bird, su tercera novela y objeto de estudio de este artículo. La historia comienza a mediados del siglo Xx, entre las ruinas del incendio de una iglesia baptista donde aparecen las memorias de Henry "Cebolla" Shackleford. En ellas, el protagonista habla de su infancia alrededor de 1850, cuando era un joven esclavo en una pequeña taberna del territorio de Kansas. Allí sirve junto a su padre hasta que un día irrumpe el Viejo John Brown, un abolicionista blanco que recorre el país en una cruzada para liberar a los negros de la esclavitud. Cebolla viaja con Brown a la costa este y a Canadá, donde el Viejo busca ayuda y se reúne con figuras clave del movimiento abolicionista negro, caso de Harriet Tubman y Fredercik Douglass, para acabar en Harpers Ferry, Virginia, donde los abolicionistas planean asaltar la armería, liberar a los esclavos del lugar y comenzar una revolución abolicionista que se propague por el país.

The Good Lord Bird explora la identidad y la supervivencia en los años precedentes al estallido de la Guerra Civil estadounidense, época que retrata con maestría y que ha granjeado a McBride notables comparaciones con Las aventuras de Huckleberry Finn, de Mark Twain (Dreisinger 2013). Ambas novelas comparten ambientación histórica, ponen de manifiesto la hipocresía de la época (Tobar 2013) y están protagonizadas por una pareja de personajes que, curiosamente, en The Good Lord Bird invierten sus roles: el blanco es el adulto y el esclavo afroamericano se convierte en el niño y en el narrador de la historia, al contrario de lo que sucedía con Jim y Huck en su travesía por el río Mississippi. El retrato que ofrece de los Estados Unidos de mediados del siglo xIX es rico y 
variopinto, pues por sus páginas desfilan esclavos negros, pistoleros del Oeste, forajidos, tropas federales, prostitutas, borrachos, líderes de la causa abolicionista y yanquis acomodados.

La novela de McBride también se inscribe en el género de las narraciones de esclavos (slave narratives). Las obras que pertenecen a este género se caracterizan por el marcado carácter abolicionista que se desprende de las historias que narran, protagonizadas por esclavos que terminan por fugarse y contar, a modo de autobiografía, los horrores de la esclavitud que presenciaron durante su cautiverio en las plantaciones del Sur de Estados Unidos. Algunos de los títulos más característicos de este género, dentro de la narrativa estadounidense, son los siguientes: Twelve Years a Slave 1 (1853), en la que Solomon Northup, un ciudadano afroamericano de los estados del Norte, describe cómo lo raptaron y su posterior cautiverio en Luisiana; y Running a Thousand Miles for Freedom; Or, The Escape of William and Ellen Craft from Slavery ${ }^{2}$ (1860), las memorias de un matrimonio negro que consiguió escapar de Georgia gracias a que la esposa, que era mulata, se hizo pasar por un terrateniente blanco que viajaba acompañado de su esclavo, quien en realidad era su marido. El género se siguió cultivando en la literatura estadounidense durante el siglo xx, en el que destacan novelas como The Confessions of Nat Turner, de William Styron (1966), dedicada al líder de una insurrección de esclavos que tuvo lugar en Virginia y fue rápidamente aplastada; y Roots ${ }^{3}$, de Alex Haley (1976), saga familiar en la que el autor rememora la vida de su antepasado Kunta Kinte, un africano al que secuestraron y llevaron a Estados Unidos.

The Good Lord Bird participa de las convenciones de este género y ofrece una versión retorcida de la historia abolicionista a través de la técnica del “manuscrito hallado”, por lo que la novela se presenta como la falsa autobiografía de Henry “Cebolla” Shackleford, un joven esclavo afroamericano que emplea, al hablar, constantes construcciones agramaticales y expresiones propias del dialecto de los negros y de las zonas rurales de Estados Unidos. Con relación a esta característica de la novela, McBride ha manifestado su interés por “el habla de los viejos negros

1. Doce años de esclavitud, traducción de Noemí Sobregués, Juan Camargo, Juan Castilla y Javier Fernández de Castro, 2013. El mismo año en el que se tradujo al español, se estrenó la adaptación cinematográfica del director Steve McQueen, que gozó del favor de la crítica y se alzó con tres premios Oscar.

2. Esta narración, escrita por el propio William Craft, no cuenta con versión en castellano.

3. Raíces, traducida por Rolando Costa Picazo, 1979. La novela inspiró una exitosa miniserie del canal ABC que se estrenó en 1977 y contó con tres secuelas acerca de los descendientes de Kunta Kinte: The Next Generation (1979), The Gift (1988) y Queen (1993). En mayo de 2016, History Channel lanzó una nueva versión de cuatro episodios basados en la obra de Haley. 
del campo, los de guitarra de blues y charla rápida, quería que pareciera un viejo echando la vista atrás y contando una gran historia” (Simon 2013). En el siguiente fragmento se puede apreciar la peculiar forma de hablar del protagonista, que presenta pasados irregulares mal conjugados (gived, knowed y thunk), construcciones agramaticales que no concuerdan en número (Colored was) y usos vulgares, como cuando emplea la forma apocopada $P a$ para referirse a John Brown:

His Pa already gived that feather to me and told me not to tell it. And I gived that feather to his son and told him not to tell. They didn't know what to believe, is how I figured it. Back in them days white folks told niggers more than they told each other, for they knowed Negroes couldn't do nothing but say, "Uh-huh,” and "Ummmm," and go on about their own troubled business. That made white folks subject to trickeration in my mind. Colored was always two steps ahead of white folks in that department, having thunk through every possibility of how to get along without being seen and making sure their lies match up with what white folks wanted. (McBride 2013: 35).

Al poco tiempo de ser publicada, The Good Lord Bird recibió numerosas críticas favorables que ensalzan su capacidad para incluir detalles históricos en la narración, a la vez que juega con las ideas de raza y género y emplea la irreverencia como homenaje (Dreisinger 2013), así como la habilidad del narrador para guiarnos por "los rincones oscuros de la historia y sugerir que las verdades pueden estar ocultas en otros lugares" (Arana 2013). La novela resulta entretenida y divertida, pero sin abandonar el tono crítico. Además, presenta a John Brown como un personaje quijotesco y a un narrador inocente y cínico, ofreciendo también "una metáfora del fallo de los blancos al reconocer la humanidad de los esclavos negros, igual que confunden a Henry con una niña” (Tobar 2013).

Una vez se han considerado las características generales de esta novela, a continuación se va a estudiar la problemática que plantea la traducción del dialecto negro o Black English en The Good Lord Bird y en el género al que pertenece, las narraciones de esclavos.

\section{El problema de la traducción del dialecto}

En el apartado anterior, se dejaba constancia de que The Good Lord Bird refleja el dialecto de los esclavos afroamericanos y de las zonas rurales de Estados Unidos, como el territorio de Kansas, donde vive el narrador al comienzo de la novela. El propio James McBride ha expresado su interés y fascinación por esta forma de hablar, que intentó recoger en la novela (Simon 2013). No obstante, 
es necesario analizar las características que definen este dialecto y qué posibles estrategias se pueden aplicar en su traducción al español.

En Traducción y traductología, Hurtado Albir habla de las variedades de la lengua que tienen que ver tanto con la persona que la utiliza como con el contexto de su uso particular (2001: 544). Esta autora distingue entre registros, que son los distintos usos de la lengua en relación con el área profesional, el nivel de formalidad o con si los textos son orales o escritos; y dialectos, que son las variedades lingüísticas que dependen de variables como el tiempo (dialecto temporal), el espacio (dialecto geográfico) o la clase social (dialecto social). De este modo, mientras que los dialectos temporales son variedades diacrónicas de la lengua, los geográficos y sociales se pueden considerar variedades diatópicas que se alejan de la lengua estándar de un determinado período.

En este sentido, la narración en primera persona de Henry “Cebolla” Shackleford es un buen ejemplo de cómo la lengua varía respecto al usuario, pues las peculiaridades de su habla quedan reflejadas en el testimonio escrito que ofrece a los lectores. El estudioso Zanger, en su artículo "Literary Dialect and Social Change” (1966: 40-48), dedicado a las particularidades con las que se ha retratado la variedad lingüística de los personajes afroamericanos en las obras literarias, define el dialecto literario como el intento de mostrar en la página impresa el habla de un grupo étnico, racial o regional concreto por medio de faltas de ortografía, omisiones, apóstrofos y cambios sintácticos. La función del dialecto literario depende de las convenciones y estereotipos que se relacionen con sus rasgos, pues puede ser objeto de burla o tan solo una marca de identidad. Zanger sostiene que sirve para caracterizar a los personajes e indicar su relación con la comunidad (que puede ser ficticia) en la que los sitúa la narración, y ofrece un recorrido por distintas modas literarias a la hora de representar el dialecto de los personajes negros: por ejemplo, distingue entre el dialecto de Guinea (caso del habla de personajes como Viernes en Robinson Crusoe), que consiste en añadir la sílaba $e e$ al final de las palabras y en cambiar la $b$ por la $v$, y el dialecto de las plantaciones. Este último se caracteriza por una estructura sintáctica y un vocabulario más complejo, además de por usos incorrectos de palabras (malapropismos ${ }^{4}$ ), algunas pronunciaciones erróneas y por la inclusión de dichos populares propios

4. El nombre de este término deviene de Mrs. Malaprop, personaje de la comedia The Rivals (1775), de Richard Brinsley Sheridan, a la que caracterizaba su habilidad para confundir palabras similares y emplearlas de forma incorrecta. Este recurso cómico se remonta a tiempos anteriores, pues ya marcaba el habla de algunos personajes de las obras de Shakespeare, por ejemplo Dogberry en Much Ado About Nothing. 
del mundo rural, como sucede con los personajes de Tom y su esposa Chloe en La cabaña del tío Tom. Määtta (2004: 320) respalda las observaciones de Zanger y señala que, dado que las variedades dialectales no siguen las convenciones de la lengua estándar, los personajes que hablan en dialecto quedan marcados y se distancian del resto. Distingue, además, cuatro técnicas para reflejar el dialecto en narrativa: las representaciones fonológicas, morfosintácticas y léxicas, y el denominado eye-dialect, que se sirve de una ortografía poco convencional para plasmar la pronunciación de los personajes con habla marcada.

Los rasgos que Zanger atribuye al dialecto de las plantaciones, así como la representación de variantes morfosintácticas y el eye-dialect que apunta Määtta, se pueden apreciar en el dialecto de Henry en The Good Lord Bird, pues la novela recoge gráficamente sus errores de pronunciación y gramaticales. Además, el protagonista utiliza participios pasados regulares para los verbos que en realidad son irregulares, recurre a formas abreviadas que marca con apóstrofos, no conjuga el verbo to be o usa la tercera persona singular is para todos los casos, y sustituye el demostrativo those por el posesivo them. Así se puede apreciar en el siguiente fragmento de la novela:

[T]hen fling hisself into a crowd of the meanest, low-down, piss-drunk Missouri rebels you ever saw. And while they mostly clubbed him to the floor and kicked out his teeth, them white fellers didn't no more blame my Pa for flinging hisself at them in the name of the Holy Ghost than if a tornado was to come along and toss him across the room, for the Spirit of the Redeemer Who Spilt His Blood was serious business out on the prairie in them days, and your basic white pioneer weren't no stranger to the notion of hope. Most of 'em was fresh out of that commodity, having come west on a notion that hadn't worked out the way it was drawed up anyway, so anything that helped them outta bed to kill off Indians and not drop dead from ague and rattlesnakes was a welcome change. It helped too that Pa made some of the best rotgut in Kansas Territory - though he was a preacher, Pa weren't against a taste or three-and like as not, the same gunslingers who tore out his hair and knocked him cold would pick him up afterward and say "Let's liquor," and the whole bunch of 'em would wander off and howl at the moon, drinking Pa's giddy sauce. Pa was right proud of his friendship with the white race, something he claimed he learned from the Bible. (McBride 2013: 8-9).

Mas Henry no es el único personaje que se expresa en dialecto en la novela, sino que el resto de esclavos y ciudadanos afroamericanos libres que aparecen en el viaje del protagonista comparten rasgos dialectales. Incluso los personajes blancos, como John Brown y su banda de forajidos abolicionistas, cometen errores gramaticales al hablar, una de las características del habla rural de Estados 
Unidos, la cual Määtta identifica con los ocasionales marcadores léxicos y morfosintácticos del dialecto que están presentes en sus intervenciones (2004: 324). En el siguiente pasaje, se puede ver cómo el capitán Brown recurre a la forma is del verbo to be de manera equívoca al dirigirse a Henry (McBride 2013: 303): "Onion, I will miss you, for you is dutiful and our Good Lord Bird besides".

Conseguir un texto castellano que tenga en cuenta estos rasgos dialectales y plasme, en castellano, la variación lingüística presente en el original es, sin duda, uno de los desafíos principales para el traductor, pues, como subraya Mateo Martínez-Bartolomé, es necesario dejar constancia en la traducción, de alguna forma, de las diferencias que presenta el Black English respecto a la variedad estándar para no perder las connotaciones sociales y culturales de este dialecto (1990: 100-101). López García respalda esta afirmación y apunta, en relación con las variedades dialectales, que "dejar de traducir esos efectos expresivos de la LO priva al lector de una información cardinal para comprender la obra que lee” (1991: 93). Por tanto, hay que "hacer llegar al lector la peculiar desviación expresiva de la norma que ofrece el dialecto", es decir, realizar un ejercicio de coherencia al trasladar las connotaciones asociadas a una variedad en particular y "explicar por qué razones [el traductor] ha elegido un determinado método de traducción” (1991: 94-95). Sin embargo, es más que habitual encontrarse con numerosas traducciones de textos afroamericanos publicadas en español que estandarizan y eliminan cualquier marca dialectal del original (como se verá en el quinto apartado de este trabajo). Este hecho no resulta sorprendente si, como asegura Landers, no hay dialecto que viaje bien en la traducción, al mismo tiempo que advierte de los peligros de usar un dialecto inventado en la lengua de llegada; de ahí su recomendación de estandarizar como norma general (1999: 116). Sáenz matiza que el problema de la traducción del dialecto sí tiene muchas soluciones, mas todas ellas resultan insatisfactorias (2000). En el siguiente apartado, se ofrecerá una relación de las varias soluciones a las que Sáenz hace referencia.

\section{Estrategias para la traducción del dialecto}

El problema, antes descrito, de la traducción de las marcas dialectales ha suscitado la atención de los traductólogos en los últimos tiempos. Tello Fons dedica su tesis doctoral (2011) y uno de sus artículos (2012) a la traducción del dialecto social y geográfico en los textos literarios, para lo cual analiza un corpus de novelas inglesas y sus correspondientes versiones en español. Defiende que la función del dialecto es mostrar la forma de hablar de una comunidad 
lingüística o establecer oposiciones entre los personajes (2011: 104) y, en ambos trabajos, resume las posturas de varios académicos con relación a la posibilidad de traducir el dialecto y las estrategias que pueden utilizarse, las cuales se verán a continuación.

En su teoría lingüística de la traducción, Catford ya define el dialecto como una variedad de la lengua que tiene que ver con la procedencia del hablante o su afiliación geográfica, temporal y social. Distingue entre registro, que es la variedad de la lengua relacionada con el rol social del hablante, e idiolecto, la variedad que emplea un individuo en particular. Sostiene que los dialectos estándares se traducirán por otro dialecto estándar de la lengua meta, mientras que no siempre será necesario ni posible traducir el idiolecto por medio de rasgos equivalentes. Asimismo, defiende la búsqueda de dialectos funcionales en la lengua de llegada o la creación de un dialecto literario para trasladar las variedades geográficas y sociales, mientras que para las temporales descarta la equivalencia total y observa que solo se puede dar al texto un "sabor arcaico" mediante la elección del léxico (1965: 83-92).

Mateo Martínez-Bartolomé, mentada en el apartado anterior, pone de ejemplo su traducción de la novela If He Hollers Let Him Go (1945), de Chester Himes, al plantear la dificultad de mantener el sabor particular del dialecto en la traducción. Con este fin, propone jugar con las variantes de la lengua de llegada, con cuidado de no caer en regionalismos excesivos, de cuyo empleo resultarían unas connotaciones incongruentes. Defiende que el traductor recurra a una variante relajada del español y emplee expresiones coloquiales y vulgares, términos sencillos, se valga del argot para mantener el tono y evite estructuras sintácticas complejas para diferenciar el lenguaje de los negros (1990: 100-104).

Rabadán también dedica un capítulo a la traducción del dialecto en su libro acerca de la equivalencia en traducción (1991), en el que, en general, adopta una postura bastante escéptica (1991: 79-109). Para ella, los dialectos representan desviaciones de la "norma culta", que se corresponde con el habla de las clases sociales más favorecidas. Sostiene que los textos que están completamente escritos en dialecto nunca deben traducirse a otro dialecto, sino a la variedad estándar de la lengua de llegada, dado su carácter uniforme, si bien se pueden añadir coletillas del tipo "dijo en dialecto". Si para mantener el sentido se depende de los rasgos dialectales, se debe elegir en la lengua meta un dialecto que pueda evocar las mismas connotaciones que el original y cumpla las mismas funciones de caracterización geográfica y social. Por otra parte, apuesta por la no traducción

5. Si grita, suéltale, traducción de Marta Mateo Martínez-Bartolomé, 1989. 
de las variantes diacrónicas, puesto que en el texto ya existen otros marcadores, en su mayoría léxicos, que lo definen temporalmente, además de que es imposible equiparar los estadios lingüísticos de ambos polisistemas (por ejemplo, se plantea a qué español sería equivalente el inglés antiguo del poema épico Beowulf, del siglo viII). Dado que el fin último, que marca la aceptabilidad de una traducción, es ser leído en el polisistema meta, en última instancia serán los lectores de la lengua de llegada quienes determinarán si la traducción del dialecto es aceptable. Según la presencia del dialecto, Rabadán distingue tres tipos de textos: los monodialectales, escritos en su totalidad en un solo dialecto; los polidialectales, en los que interviene más de una variedad lingüística; y los textos parcialmente monodialectales, en los que los lectores entran en contacto con un dialecto determinado a través de uno o más personajes.

Muñoz Martín también deja de lado el dialecto temporal y se centra en la traducción de los dialectos geográficos y sociales (1995: 5-42). Mantiene que, si los rasgos dialectales son intencionados por parte del autor del texto de origen, ejercen una función concreta que debe reflejarse en la traducción, por lo que "es necesario asignarles un significado, para lo que se recurre a la gama de estereotipos de la comunidad lingüística en cuyo seno se crean”. Por otra parte, si los rasgos dialectales reflejan el idiolecto de los emisores de forma no intencionada, no hay por qué mantenerlos en la traducción.

Hatim y Mason (1995: 55-64) ponen de manifiesto el problema insoslayable de qué dialecto de la lengua de llegada hay que usar en la traducción, puesto que, si se traduce por la norma culta estándar de la lengua meta, se pierden los efectos especiales pretendidos por el texto fuente; mientras que si se traduce un dialecto por otro existe el riesgo de crear efectos distintos de los buscados originariamente. Destacan los problemas de comprensión, incluso para los lectores de los textos de la lengua fuente, que suponen los dialectos temporales, para los cuales proponen dos opciones: la traducción hacia una variante arcaica de la lengua de llegada o hacia la variante estándar y contemporánea. En cuanto a los dialectos sociales y geográficos, Hatim y Mason subrayan su función sociocultural y la relevancia de las implicaciones políticas e ideológicas que puedan conllevar, pues los dialectos son una consecuencia de la estratificación social en el seno de la comunidad lingüística. Inciden en la necesidad de traducirlos y de respetar toda la carga social del original, pero no dicen cómo hacerlo, si bien apuntan que no es necesario seleccionar una variedad regional en concreto, sino que se puede recurrir a modificaciones del estándar, por ejemplo a variar la gramática y el léxico de la lengua de llegada de forma deliberada. 
Mayoral Asensio, por su parte, asegura, al igual que Sáenz, que la traducción del dialecto social o geográfico es un problema sin solución satisfactoria y que es inevitable perder parte del contenido en el proceso. Considera seis opciones distintas (1990: 35-46): la traducción al estándar de la lengua de llegada, que implica una gran pérdida de contenido pero no produce efectos no deseados en los lectores; la traducción a otro dialecto de la lengua de llegada, que puede producir incredulidad y comicidad en los lectores; la traducción a variedades subestándar de la lengua de llegada, de forma que se pueda mantener el tono, si bien puede chocar con el habla de personajes cultos y con las situaciones formales; la traducción a variedades idiomáticas, así se mantiene el tono, pero se pierde información, además de que la caracterización de los personajes se resiente; y el uso de elementos fonéticos que el lector de la lengua de llegada identifica con el origen que marca el texto fuente. Da los ejemplos de sustituir la $r$ por la $l$ para personajes chinos o la $r$ por la $g$ para los franceses, pero son clichés y estereotipos bastante descarados. En el caso del dialecto afroamericano, propone el ejemplo de los personajes negros que hablan con acento cubano, como en el doblaje castellano de Lo que el viento se llevó. Por último, Mayoral Asensio también plantea usar elementos léxicos o sintácticos que el lector de la lengua meta identifique con el origen que marca la lengua fuente. Por ejemplo, hablar como los indios (con infinitivos y sin determinantes) o imitar el orden sintáctico alemán con fines cómicos.

En su trabajo posterior de 1999, este autor se muestra contrario a la traducción por dialectos funcionales debido al choque cultural que pueden provocar en el lector, y considera las expectativas de los participantes en cada situación comunicativa. Con ellas en mente, Mayoral Asensio propone nueve máximas para traducir el dialecto de forma eficaz (1999: 170-172), que son ajustarse al contexto y la situación, ajustar la estrategia comunicativa al encargo de la traducción, utilizar solo marcadores con los que esté familiarizado el lector, mantener solo las distinciones que el lector pueda apreciar, no mantener en el texto meta las distinciones del texto original que no tengan una función comunicativa, utilizar el mínimo de marcadores que permita reproducir el efecto deseado, no introducir ambigüedad injustificada, evitar la incoherencia, por ejemplo, no mezclar rasgos propios de las culturas fuente y meta, y mantener la coherencia en el tipo de marcadores utilizados para señalar un rasgo determinado y el conjunto de los rasgos de un texto.

A propósito de la traducción del dialecto, la ya mencionada Hurtado Albir (2001: 583) advierte de que hay que identificar la función que cumplen las formas no estándar en la obra original y marcarlas en la traducción. También apuesta por una solución dinámica para la traducción del dialecto geográfico, del social 
e incluso del temporal, que tenga en cuenta las características de la cultura meta y sus usos lingüísticos y se adapte a ellos. Finalmente, los rasgos idiolectales son especialmente relevantes en textos de un alto grado de autoría, por lo que el traductor debe detectar y reproducir estas marcas para que el texto meta también genere autoría y peculiaridad. Puesto que el uso del dialecto en narrativa ayuda a diferenciar a qué clase social pertenecen los personajes, el mentado Määtta distingue, en la línea de Hurtado Albir, tres estrategias predominantes para la traducción del dialecto, que son la estandarización, la inclusión de léxico coloquial, y una combinación de estas dos, es decir, un tipo de estandarización que permita recurrir ocasionalmente al léxico de corte vulgar para sugerir la clase social del hablante (2004: 323-325).

Aun sin ser el objeto de estudio de este artículo, el teatro tampoco es ajeno al problema que suscita traducir el dialecto, que Zatlin sitúa en lo más alto de la lista de desafíos a los que un traductor, en este caso dramático, puede enfrentarse (2005: 82-84). Con el fin de respetar los matices y las implicaciones del uso del dialecto en el texto fuente, esta autora remite a las estrategias que desarrolló Perteghella a partir de las traducciones al alemán y al italiano de las obras de Edward Bond y de George Bernard Shaw. Estas cinco estrategias son, de forma resumida, las siguientes cinco (2002: 50-51): la compilación dialectal, que mantiene la ambientación original y ofrece una mezcla de modismos o dialectos distintos de la lengua meta; la traducción pseudodialectal, de forma que los nombres y las referencias culturales del original también se mantienen y se traduce por un dialecto ficticio que no es ninguno específico, pero sí es accesible para la gran mayoría de los lectores; la traducción dialectal paralela, en la que tampoco se alteran los nombres ni las referencias culturales del texto fuente, pero se traduce por un dialecto concreto de la cultura de llegada que despierte las mismas connotaciones y tenga una posición similar en ella; la localización dialectal, que es un caso de aculturación, puesto que se domestica todo el texto, incluidos los nombres de los personajes y lugares, las referencias culturales y los dialectos; y, por último, la mencionada estandarización o nivelación, mediante la cual se eliminan todas las variaciones dialectales, con la excepción de coloquialismos ocasionales. Aunque la estandarización garantiza la comprensión del texto de llegada, se pierden la fuerza y los matices del dialecto en el texto original. Perteghella concluye con la recomendación de evaluar la distancia entre la cultura fuente y la meta antes de decidirse por aplicar una estrategia concreta, y sugiere tomar una vía intermedia que no traicione la cultura fuente ni tampoco haga que el texto traducido sea ilegible. 
Volviendo a Tello Fons, esta autora defiende que los traductores deben tener la posibilidad de llevar a cabo la traducción de la variación lingüística y de elegir entre un abanico de opciones según la función del dialecto en el texto fuente. Considera la variación de la norma lingüística de la lengua meta como un vehículo para dar credibilidad a un dialecto que en el original reflejaba el habla de un lugar, grupo social o personaje determinado (2011: 117). También contempla la posibilidad de crear un dialecto propio para el personaje en cuestión e intentar así que su habla lo caracterice, si bien reconoce el riesgo de caer en estereotipos, construcciones demasiado artificiales y de que, a pesar de estos esfuerzos, los lectores asocien ese dialecto a una forma de hablar ya existente. Puntualiza que no se trata de reproducir el dialecto como tal, sino sus evocaciones e implicaciones, ya que "los hablantes de cada comunidad otorgamos una serie de valores a los dialectos y a las personas que los hablan que tienen sentido precisamente dentro de esa comunidad o contexto social” (2011: 120). Reconoce que la traducción del dialecto entraña un riesgo en la aceptabilidad del texto meta, pues, en última instancia, son los lectores quienes deciden si la traducción funciona. Aparte de los lectores, la aceptabilidad de la traducción del dialecto también depende de los requisitos del encargo de la traducción, de su finalidad y del estatus de la literatura traducida en el polisistema de la cultura de llegada.

Tello Fons considera que la presencia del dialecto en una obra literaria puede tener dos funciones, compatibles entre sí (2011: 140-165): la función mimética, que refleja la realidad del habla de una comunidad lingüística determinada; y la función simbólica, en la que el dialecto actúa de herramienta para establecer oposiciones entre personajes, proyecta una ideología determinada y busca mover los sentimientos de los lectores hacia los personajes. Al dotar a un personaje de un habla dialectal, el autor lo está destacando. La autora también subraya que las marcas dialectales requieren un esfuerzo extra de comprensión por parte de los lectores y que "en cualquier dialecto se encuentran rasgos de todos los demás, pero siempre habrá uno que tenga protagonismo [...] tras un dialecto geográfico puede descubrirse una intención de plasmación de una realidad social” (2011: 147-148).

En cuanto a las soluciones que se pueden dar para la traducción de las variedades dialectales, Tello Fons adopta la propuesta de Marco Borillo (2002: 80-86) y se plantea, en primer lugar, optar por un texto con marcas, que transgreda la norma lingüística de la lengua meta en alguno de sus niveles (ortográfico, gramatical o léxico) o por uno sin marcas, estandarizado. En segundo lugar, existe la posibilidad de elegir entre un dialecto en concreto de la lengua de llegada para la traducción y una configuración artificial que mezcle rasgos de varios dialectos y no recuerde a ninguno específico. La autora también se sirve de la estilística 
para estudiar el texto fuente desde un enfoque lingüístico y de forma rigurosa y sistemática. Sostiene que el traductor debe tener en cuenta la función caracterizadora del diálogo y del dialecto que emplean los personajes, la ideología y el valor estético detrás de estos rasgos, al igual que las expectativas de los lectores de la cultura meta, para ofrecer una solución que pueda equiparar las connotaciones del dialecto original a través de unos marcadores que las intenten reproducir con la máxima fidelidad posible, buscando siempre la coherencia del texto en la lengua de llegada.

Más recientemente, y a propósito de este tema particular, Paradela López (2014) rescata las soluciones de corte funcionalista propuestas por Marco Borillo y Tello Fons y vuelve a subrayar la necesidad de que no se pierdan las connotaciones del original. Distingue entre el anatopismo, que se corresponde con la localización dialectal y hace creer a los lectores, por ejemplo, que los personajes son gallegos o andaluces por su forma de hablar (los regionalismos excesivos de los que ya advertía Mateo Martínez-Bartolomé), aunque la novela esté ambientada en otro lugar; y el atopismo, que equivale a la compilación dialectal y consiste en crear dialectos que no se correspondan con ningún lugar concreto, si bien su presencia hace que el lector note una diferencia en el habla. Antolín Rato (2014) describe cómo suelen desaparecer las connotaciones sociales del Black English al estandarizarlo en español o, peor aún, al traducirlo por un acento regional hispano. De ahí que anime a los traductores a que no se pierda el sentido de pertenencia a una comunidad concreta que tiene para los negros el dialecto, que los diferencia de los personajes blancos y les permite identificarse con los miembros de su etnia, con quienes comparten una variedad lingüística particular.

Una vez se han considerado las distintas estrategias para la traducción del dialecto literario, en el siguiente apartado se observa cuáles predominan en las traducciones al castellano de las narraciones de esclavos.

\section{El dialecto en las narraciones de esclavos: casos prácticos}

Tras repasar estas consideraciones teóricas, las siguientes líneas se centrarán en la práctica de la traducción del Black English, la variedad que prima en la novela The Good Lord Bird, y se observarán las estrategias que se utilizan. Con este propósito, a continuación se presenta una muestra de cuatro novelas estadounidenses, y de sus traducciones al español, que comparten la presencia del dialecto afroamericano en el habla de los esclavos: La cabaña del tío Tom, 
de Harriet Beecher Stowe; Las aventuras de Huckleberry Finn, de Mark Twain; Raíces, de Alex Haley; y Beloved, de Toni Morrison.

En La cabaña del tío Tom, obra de marcado carácter abolicionista, quedan reflejadas las particularidades de la forma de hablar de los esclavos por medio del eye-dialect o transcripción de la pronunciación de las palabras, lo cual se puede apreciar en la siguiente intervención de la tía Chloe, la esposa de Tom (Stowe 1986: 72): “And so ye telled Tom, did ye? O, Lor! What young uns will be up ter! Ye crowed over Tom? O, Lor! Mas'r George, if ye wouldn’t make a hornbug laugh!”. Sin embargo, la traducción al español de Vázquez de Castro recurre a la estandarización y suprime cualquier marca dialectal: “¿Así que eso le dijo usted a Tom, verdad? ¡Dios mío, qué jóvenes éstos, qué cosas se les ocurren! ¿Cacarearle a Tom? Dios mío, señorito, sería usted capaz de hacer reír a un muerto” (Stowe 1992: 32).

En el caso de Las aventuras de Huckleberry Finn, no solo está presente el dialecto afroamericano, que marca el habla de Jim, un esclavo fugado, sino que al joven y célebre protagonista también lo caracterizan ciertos dejes del habla rural de Estados Unidos:

'Hallo, Jim!' and skipped out.

He bounced up and stared at me wild. Then he drops down on his knees, and puts his hands together and says:

'Doan' hurt me - don't! I hain't ever done no harm to a ghos'. I awluz liked dead people, en done all I could for 'em. You go en git in de river agin, whah you b’longs, en doan' do nuffn to Ole Jim, 'at 'uz awluz yo' fren'.' (Twain 2001: 199).

Como en el caso anterior, la técnica elegida para traducir este texto al castellano ha sido la nivelación, que garantiza la comprensión del texto meta pero elimina irremediablemente los matices presentes en el original, así como los distintos registros que emplean los dos protagonistas:

— ¡Hola, Jim! —y salí de un brinco.

Él se puso de pie de un salto y me miró con ojos de loco. Luego cayó de rodillas y juntó las manos y dijo:

— ¡No me hagas daño, no! Nunca he hecho daño a un fantasma. Siempre me gustaban los muertos y les he hecho todo el bien que pude. Vete y métete en el río otra vez, donde debes estar, y no le hagas nada al viejo Jim, que siempre fue buen amigo. (Twain 2004: 56). 
A este respecto, Julià Ballbè (1997: 196-199) subraya la dificultad añadida de traducir el habla rural de los blancos y diferenciarla del dialecto de los negros. Distingue, asimismo, dos grupos de las traducciones de Huckleberry Finn al castellano y al catalán, las que se proponen reflejar, de alguna manera, la singularidad del habla de Jim y los negros y las que ni siquiera se lo plantean y recurren a la estandarización. Este autor también hace hincapié en el escollo que supone la nota introductoria de la novela, en la que Twain afirma que ha reflejado en su obra hasta siete dialectos regionales diferentes (2001: 167). Aunque Julià Ballbè defiende la función caracterizadora de los dialectos en la novela de Twain, reconoce la dificultad añadida que suponen para los traductores, quienes incluso optan por eliminar la mentada nota introductoria o por complementarla con una nota del traductor que expliqué qué estrategias se han empleado (1997: 199-200).

En el caso de las narraciones de esclavitud del siglo Xx (neo-slave narratives) que retratan el dialecto de los protagonistas, la tendencia traductora sigue siendo la misma: eliminar cualquier marca de variedad lingüística y ofrecer un texto en castellano estándar. Así se aprecia en el siguiente fragmento, perteneciente al final del capítulo cincuenta y seis de Raíces, cuando un grupo de esclavos se entera de las noticias del final de la Guerra de la Independencia de Estados Unidos y se pregunta qué consecuencias puede tener para su libertad:

“Y'all come here!” Bell shouted, summoning the others on slave row not long after. "Massa jes' tol' me dey done named that Philadelphia firs' capital of Newnited States!” But it was Luther who told them later, "Massa Jefferson done put up some kin' of Manumission Ack. It say massas got de right to free niggers, but tell me dem Quakers an' antislavery folks an' free niggers up Nawth is hollerin' an' goin' on 'cause the Ack say massas don't have to, not less'n dey wants to." (Haley 2006: 206).

La versión española de Costa Picazo no conserva ningún rastro de las marcas dialectales del texto original y del eye-dialect:

—iVenid todos aquí! — gritó Bell al poco rato—. El amo me acaba de decir que han designado a Filadelfia como capital de los Estados Unidos.

Pero fue Luther quien les dijo después:

—El amo Jefferson ha aprobado una ley de manumisión. Según ella, los amos tienen el derecho de dar la libertad a los esclavos, pero cuentan que los cuáqueros, los que están en contra de la esclavitud y los negros liberados del Norte están enojados porque la ley dice que los amos no están obligados a darles la libertad a no ser que lo deseen. (Haley 1979: 218). 
Lo mismo sucede con la traducción al español de Beloved, la novela de Morrison acerca de la maternidad y los fantasmas de la esclavitud en la era de Reconstrucción. En el siguiente fragmento se pueden apreciar las variaciones de carácter morfosintáctico del habla de Sethe cuando recuerda su infancia en la plantación (Morrison 1988a: 60): "My woman? You mean my mother? If she did, I don't remember. I didn't see her but a few times out in the fields and once when she was working indigo. She must of nursed me two or three weeks". Al igual que en los ejemplos anteriores, la estrategia empleada por la traductora ha sido la estandarización y eliminación de cualquier marca dialectal: “¿Mi mujer? ¿Te refieres a mi madre? Si lo hizo, no me acuerdo. Sólo la vi unas veces, en el campo, y un día que estaba haciendo tintes. Debió de darme la teta dos o tres semanas” (Morrison 1988b: 74). A propósito del predominio de la estandarización, Bernárdez Sanchíz apunta a la preferencia de las editoriales por la uniformidad, la simplificación y la eliminación de las diferencias en la lengua escrita, de modo que no se produzcan cambios de registro, con el fin de llegar al mayor número de lectores posible (2012: 103-111).

La siguiente sección ofrece una propuesta de traducción alternativa a la estandarización que tiene en cuenta las consideraciones expuestas en el cuarto apartado.

\section{La traducción del dialecto en The Good Lord Bird}

Como queda dicho en el segundo apartado de este artículo, en la novela de McBride el narrador plasma sus desviaciones del Standard English, tanto de pronunciación como de gramática, características del inglés de los esclavos, al escribir y rememorar sus aventuras junto a John Brown, al mismo tiempo que refleja las incorrecciones del habla rural de los personajes blancos. Una traducción neutralizada, como los casos de las versiones castellanas de las narraciones de esclavos que se han visto en el apartado cinco, daría el resultado que se expone a continuación, ejemplificado en este fragmento de The Good Lord Bird, correspondiente al que figura en el tercer apartado de este artículo:

[T] hen fling hisself into a crowd of the meanest, low-down, piss-drunk Missouri rebels you ever saw. And while they mostly clubbed him to the floor and kicked out his teeth, them white fellers didn't no more blame my Pa for flinging hisself at them in the name of the Holy Ghost than if a tornado was to come along and toss him across the room, for the Spirit of the Redeemer Who Spilt His Blood was serious business out on the prairie in them days, and your 
basic white pioneer weren't no stranger to the notion of hope. Most of 'em was fresh out of that commodity, having come west on a notion that hadn't worked out the way it was drawed up anyway, so anything that helped them outta bed to kill off Indians and not drop dead from ague and rattlesnakes was a welcome change. It helped too that Pa made some of the best rotgut in Kansas Territory-though he was a preacher, Pa weren't against a taste or three-and like as not, the same gunslingers who tore out his hair and knocked him cold would pick him up afterward and say "Let's liquor," and the whole bunch of 'em would wander off and howl at the moon, drinking Pa's giddy sauce. Pa was right proud of his friendship with the white race, something he claimed he learned from the Bible. (McBride 2013: 8-9).

Luego se tiraba encima de una multitud de la peor escoria, la de los rebeldes de Misuri más borrachuzos que jamás hayáis visto. La mayoría lo golpeaba hasta tumbarle en el suelo y sacarle los dientes a patadas, pero a esos tipos blancos les daba igual que mi padre se los echase encima en el nombre del Espíritu Santo o que viniera un tornado y lo mandase volando de un lado a otro de la taberna; en aquellos tiempos el Espíritu del Redentor Que Derramó Su Sangre por Nosotros era un asunto serio en la pradera, y a los típicos pioneros blancos no les era ajeno el concepto de la esperanza. Casi todos lo tenían bien reciente al haber venido al oeste con una idea que no había salido como se esperaban, así que daban la bienvenida a cualquier cambio que los ayudara a levantarse de la cama para matar indios y no morirse por culpa de las fiebres o de las serpientes de cascabel. También ayudaba que mi padre hacía el mejor aguardiente del territorio de Kansas (a pesar de que era predicador, no le molestaba tomar un trago, o tres) y, aunque parezca mentira, los mismos pistoleros que le arrancaban el pelo a tirones y le daban una buena tunda solían levantarlo después y decir: «Vamos a beber»; y todos juntos se marchaban a aullar a la luna mientras bebían el licor de la felicidad de mi padre. Se sentía orgulloso de su amistad con la raza blanca; la había aprendido de la Biblia, o eso decía.

No obstante, esta posibilidad no respeta las observaciones, antes expuestas, de Mateo Martínez-Bartolomé (1990) ni de López García (1991) y se olvida de trasladar a los lectores la peculiar desviación de la norma presente en la obra y fundamental para su comprensión. Además, implica una gran pérdida del contenido y los matices del texto fuente, como señalaba Mayoral Asensio (1990). De ahí que, en mi propuesta de traducción de la novela, se sigan las nueve máximas de adecuación y coherencia que exponía este autor y las recomendaciones de Tello Fons (2011) y se opte por un texto en español con marcas que transgredan la norma lingüística con el fin de respetar las funciones mimética y simbólica de la variedad que recoge el texto fuente. Para evitar el anatopismo, la domes- 
ticación y que a los personajes de la novela se los asocie con las connotaciones de un dialecto español específico, peligro del que advertía Paradela López, se aplica la estrategia que Perteghella llamaba "compilación dialectal”, de modo que se mantiene la ambientación del texto original y se traduce el dialecto de los personajes por una mezcla de modismos y distintos rasgos dialectales de la lengua de llegada. En esta traducción, los personajes de The Good Lord Bird que emplean el dialecto afroamericano pasan a presentar una compilación de rasgos propios del español coloquial y vulgar, como por ejemplo la pérdida de la $d$ de los participios (venío) y de final de palabra (esclavitú), la pérdida de las vocales de las conjunciones ( $q u$ '), preposiciones ( $d^{\prime}$ ') y pronombres personales ( $\left(l^{\prime}\right)$ cuando la siguiente palabra comienza por vocal, el leísmo (le mandase), el empleo de vosotros cuando el narrador se dirige a los lectores y el uso de las formas vulgares apocopadas pa, na, mu y to, en lugar de para, nada, muy y todo, respectivamente. De este modo, el fragmento anterior, lejos de permanecer estandarizado, presenta la forma que se recoge a continuación al seguir esta estrategia:

Luego se tiraba encima d'una multitú de la peor escoria, la de los rebeldes de Misuri más borrachuzos que jamás hayáis visto. La mayoría le golpeaba hasta tumbarle en el suelo y sacarle los dientes a patás, pero a esos tipos blancos les daba igual que mi papa se los echase encima en el nombre del Espíritu Santo o que viniera un tornao y le mandase volando d'un lao a otro de la taberna; en aquellos tiempos el Espíritu del Redentor Que Derramó Su Sangre por Nosotros era un asunto serio en la pradera, y a los típicos pioneros blancos no les era ajeno el conceto de la esperanza. Casi tos lo tenían bien reciente al haber venío al oeste con una idea que no había salío como s’esperaban, así que daban la bienvenía a cualquier cambio que los ayudara a levantarse de la cama pa matar indios y no morirse por culpa de las fiebres o de las serpientes de cascabel. También ayudaba que papa hacía el mejor aguardiente del territorio de Kansas (a pesar de qu'era predicador, a papa no le molestaba tomar un trago, o tres) y, aunque parezca mentira, los mismos pistoleros que l'arrancaban el pelo a tirones y le daban una buena tunda solían levantarle después y decir: «Vamos a beber»; y tos juntos se marchaban a aullar a la luna mientras bebían el licor de la felicidá de papa. Se sentía orgulloso de su amistá con la raza blanca; l’había aprendío de la Biblia, o eso decía. (McBride 2017: 24-25).

Con la estrategia seguida en la traducción de este extracto, que se seguirá en el resto de la novela, se logra que no solo se respeten, en el texto meta, parte de las connotaciones del Black English que utilizan los esclavos y que ponen de manifiesto su bajo nivel de educación, sino que además se los identifique como una comunidad de hablantes que comparten una variedad lingüística concreta. Sin 
embargo, dado que la novela es un texto polidialectal, una de las limitaciones de esta traducción es la dificultad de buscar otros rasgos dialectales en la lengua de llegada que reflejen las particularidades del habla rural de los personajes blancos y los diferencien del resto. Si bien una posible apuesta sería la compilación dialectal, de modo que sus intervenciones presentaran una menor cantidad de rasgos propios del español vulgar que los diálogos de los personajes afroamericanos, esta no resulta convincente por la confusión que se generaría respecto a qué personajes hablan en un dialecto determinado y cuáles no. Finalmente, en esta propuesta se ha recurrido a estandarizar el habla de los blancos para contrastarla con el Black English de los esclavos, con la consecuente desaparición de algunos matices. Esta pérdida se aprecia, por ejemplo, en el siguiente fragmento, cuando Henry “el Holandés” Sherman, el dueño del protagonista, se expresa así:

"I'm gonna make you swear on this Bible that you is for slavery and the U.S. Constitution," he said. "If you do that, you old bag, you can walk outta here none the worse. But if you're a lying, bluebellied Free Stater, I'mma bust you across the head so hard with this pistol, yellow'll come out your ears. Place your hand on that." (McBride 2013: 15).

Como se observa en la propuesta traducción al castellano, la neutralización de las marcas dialectales hace que el pasaje se lea del siguiente modo:

— Voy a hacer que jures sobre esta Biblia que estás a favor de la esclavitud y de la Constitución de los Estados Unidos — dijo- - Si lo juras, vejestorio, te puedes ir de aquí sin que pase nada; pero si eres un embustero cagueta en contra de la esclavitud, te voy a atizar en la cabeza con esta pistola tan fuerte que se te va a salir el cerebro por las orejas. Pon la mano aquí. (McBride 2017: 24-25).

Esta diferenciación del dialecto de los blancos se ajusta a las recomendaciones de Määtta, quien sugiere que aquellos pasajes con dialecto literario menos marcado se estandaricen para que así contrasten con aquellos dialectos más marcados que contribuyen a a construcción del Otro en la novela, caso del Black English (2004: 332-335).

\section{Conclusiones}

Como se ha visto en este artículo, la traducción del dialecto en las narraciones de esclavos plantea un problema difícil y, aunque se cuenta con varias 
estrategias posibles para afrontarlo, resulta harto complicado mantener el sabor particular del dialecto y sus connotaciones sociales y culturales en la lengua de llegada. Es más, los ejemplos que se han analizado muestran el predominio de la estandarización y la pérdida de las diferencias sociales y culturales que implican los distintos dialectos presentes en las novelas originales. No obstante, es responsabilidad del traductor dejar constancia de estas diferencias, relevantes para el retrato de los personajes y la comunidad a la que pertenecen.

La propuesta de traducción al castellano de la novela The Good Lord Bird se guía por la observación de López García (1991) de hacer transmitir la desviación expresiva del texto fuente a los lectores de la lengua de llegada, aunque se recurre a la estandarización en el caso concreto del habla rural de los personajes blancos, como aconsejaba Määtta (2004). Para que no se pierda la función sociocultural del dialecto afroamericano en la novela ni sus evocaciones e implicaciones, de las que hablaban Hatim y Mason (1995), se opta por traducir el Black English a una variedad subestándar de la lengua meta siguiendo las nueve máximas de adecuación y coherencia que exponía Mayoral Asensio (1999). Así, la estrategia seleccionada es la que Perteghella definía como "compilación dialectal” (2002), a la que a su vez se referían Tello Fons (2011) y Marco Borillo (2002) al considerar la posibilidad de traducir por un texto con marcas que transgredan la norma lingüística, es decir, una configuración artificial que mezcle rasgos de varios dialectos, en concreto del español coloquial y vulgar, como propone Mateo Martínez-Bartolomé (1990), y que tenga en cuenta las funciones mimética y simbólica del dialecto en The Good Lord Bird. De este modo, y según la terminología de Paradela López (2014), se opta por el atopismo frente a la localización dialectal o anatopismo.

En futuros estudios, se investigará cómo la elección de determinadas estrategias traductoras transforma, ideologiza y configura la identidad de los esclavos, la comunidad asociada al dialecto. La traducción de un texto que representa al Otro supone un reto particular, pues no solo se reconfigura su identidad en la cultura de llegada, sino que también se construye y proyecta la identidad del traductor según la ideología predominante o los intereses comerciales preponderantes (Braga Riera 2011: 67; Martín Ruano y Vidal Sales 2013: 89-90).

\section{Bibliografía}

ANTOLÍN RATO, M. (2014). "Eñorita Ecalaata” <http://cvc.cervantes.es/trujaman/anteriores/abril_14/25042014.htm>. (Acceso 28 Marzo 2016). 
ARANA, M. (2013). “The Good Lord Bird’ by James McBride” < http://www. washingtonpost.com/entertainment/books/the-good-lord-bird-by-james-mcbride/2013/08/19/e0759a98-05e1-11e3-88d6-d5795fab4637_story.html>. (Acceso 30 Marzo 2016).

BRAGA RIERA, J. (2011). “¿Traducción, adaptación o versión?: maremágnum terminológico en el ámbito de la traducción dramática”. Estudios de Traducción 1: 59-72.

BERNÁRDEZ SANCHÍS, E. (2012). “Traduttore-traditore... ¿ ¿o editore-destruttore?” en Lengua, traducción, recepción: en honor de Julio César Santoyo. (Eds. R. Rabadán et al.). León: Universidad de León.

CATFORD, J. C. (1965). A Linguistic Theory of Translation. Londres: Oxford University Press.

CRAFT, W. (2004). Running a Thousand Miles for Freedom; Or, The Escape of William and Ellen Craft from Slavery. <http://docsouth.unc.edu/neh/craft/ menu.html>. (Acceso 6 mayo 2015).

DREISINGER, B. (2013). “Marching On: James McBride’s 'Good Lord Bird”” <http://www.nytimes.com/2013/08/18/books/review/james-mcbrides-goodlord-bird.html>. (Acceso 30 Marzo 2016).

HALEY, A. (1979). Raíces. Madrid: Ultramar Ediciones. Trad. R. Costa Picazo. HALEY, A. (2006). Roots. Nueva York: Vanguard Press.

HATIM, B. y MASON, I. (1995). Teoría de la traducción: Una aproximación al discurso. Barcelona: Ariel. Trad. S. Peña.

HIMES, C. (1986). If He Hollers Let Him Go. Nueva York: Thunder's Mouth Press.

HIMES, C. (1989). Si grita, suéltale. Gijón: Ediciones Júcar. Trad. M. Mateo Martínez-Bartolomé.

HURTADO ALBIR, A. (2001). Traducción y traductología: Introducción a la traductología. Madrid: Cátedra.

JULIÀ BALLBÈ, J. (1997). “The Adventures of Huckleberry Finn i les traduccions impossibles” en Traducció i literatura: Homenatge a Ángel Crespo. (Eds. S. González Ródenas y F. Lafarga). Vic: Eumo editorial.

LANDERS, C. E. (1999). Literary Translation: A Practical Guide. Clevedon Buffalo - Toronto: Multilingual Matters Ltd.

LÓPEZ GARCÍA, D. (1991). Sobre la imposibilidad de la traducción. Cuenca: Universidad de Castilla-La Mancha.

MÄ̈̈TTA, S. K. (2004). "Dialect and point of view: The ideology of translation in The Sound and the Fury in French”. Target 16 (2): 319-339.

MARCO BORILlO, J. (2002). El fil d’Ariadna: Anàlisi estilística i traducció literària. Barcelona: Eumo. 
MARTÍN RUANO, M. R. y VIDAL SALES, C. (2013). "Identidades en traducción: representaciones transculturales de las subjetividades colectivas en los medios”. Debats 121: 84-93.

MATEO MARTÍNEZ-BARTOLOMÉ, M. (1990). "La traducción del Black English y el argot negro norteamericano". Revista alicantina de Estudios Ingleses 3: 97-106.

MAYORAL ASENSIO, R. (1990). "Comentario a la traducción de algunas variedades de la lengua”. Sendebar 1: 35-46.

MAYORAL ASENSIO, R. (1999). La traducción de la variación lingüística. Soria: Diputación Provincial.

MCBRIDE, J. (2013). The Good Lord Bird. Nueva York: Riverhead Books.

MCBRIDE, J. (2017): El pájaro carpintero. Gijón: Hoja de Lata. Trad. M. Sanz Jiménez.

MORRISON, T. (1988a). Beloved. Londres: Picador.

MORRISON, T. (1988b). Beloved. Barcelona: Círculo de Lectores. Trad. I. Menéndez Sallés.

MUÑOZ MARTÍN, R. (1995). Lingüística para traducir. Barcelona: Teide.

NORTHUP, S. (2013). Doce años de esclavitud. Barcelona: Debolsillo. Trad. N. Sobregués, J. Camargo, J. Castilla y J. Fernández de Castro.

NORTHUP, S. (2014). Twelve Years a Slave. Londres: Collins Classics.

PARADELA LÓPEZ, D. (2014). “Traducir Dialectos”. <http://cvc.cervantes.es/ trujaman/busqueda/resultadosbusqueda.asp?vve $=50 \&$ pagina $=1 \&$ titulo=tradu cir\%20dialectos\%20\&ordenresultador=2>. (Acceso 28 marzo 2016).

PERTEGHELlA, M. (2002). "Language and Politics on Stage: Strategies for Translating Dialect and Slang with References to Shaw's Pygmalion and Bond's Saved". Translation Review 64: 45-53.

RABADÁN, R. (1991). Equivalencia y traducción: Problemática de la equivalencia translémica inglés-español. León: Universidad de León.

SÁENZ, M. (2000). "Dialectos dilectos". <http://cvc.cervantes.es/trujaman/anteriores/noviembre_00/03112000.htm>. (Acceso 28 Marzo 2016).

SIMON, S. (2013). “'Good Lord Bird' Gives Abolitionist Heroes Novel Treatment” <http://www.npr.org/2013/08/17/212588754/good-lord-bird-gives-abolitionist-heroes-novel-treatment>. (Acceso 30 Marzo 2016).

STOWE, H. B. (1986). Uncle Tom's Cabin. Nueva York: Penguin.

STOWE, H. B. (1992). La cabaña del tío Tom. Madrid: Anaya. Trad. I. Vázquez de Castro.

STYRON, W. (2004). The Confessions of Nat Turner. Londres: Vintage Books. TELLO FONS, I. (2011). La traducción del dialecto: análisis descriptivo del dialecto geográfico y social en un corpus de novelas en lengua inglesa y su traducción al español. Tesis doctoral inédita, Universitat Jaume I. 
TELLO FONS, I. (2012). “Traducción de la variación lingüística: Una visión diacrónica”. Hikma 11: 133-159.

TOBAR, H. (2013). “'The Good Lord Bird' Is a Twisted Take on an Abolitionist’s Story” <http://articles.latimes.com/2013/aug/30/entertainment/la-ca-jcjames-mcbride-20130901>. (Acceso 30 Marzo 2016).

TWAIN, M. (2001). The Adventures of Tom Sawyer \& The Adventures of Huckleberry Finn. Ware: Wordsworth Classics.

TWAIN, M. (2004). Las aventuras de Huckleberry Finn. Madrid: El País. Trad. D. Rolfe y A. Ferres.

ZANGER, J. (1966). "Literary Dialect and Social Change”. Midcontinent American Studies Journal 7 (2): 40-48.

ZATLIN, P. (2005). Theatrical Translation and Film Adaptation: A Practitioner's View. Clevedon - Buffalo - Toronto: Multilingual Matters Ltd. 